\title{
Long-term glucose variability and risk of nephropathy complication in UKPDS, ACCORD and VADT trials
}

\author{
Jin J. Zhou ${ }^{1,2}$ (D) $\cdot$ Ruth Coleman ${ }^{3} \cdot$ Rury R. Holman ${ }^{3} \cdot$ Peter Reaven $^{2}$ \\ Received: 27 May 2020 / Accepted: 29 July 2020 / Published online: 21 September 2020 \\ (C) Springer-Verlag GmbH Germany, part of Springer Nature 2020
}

Keywords Intensive glycaemic control $\cdot$ Long-term glycaemic variability $\cdot$ Microvascular complications $\cdot$ Nephropathy $\cdot$ Type 2 Diabetes

\begin{tabular}{ll}
\multicolumn{2}{l}{ Abbreviations } \\
ACCORD & $\begin{array}{l}\text { Action to Control Cardiovascular Risk in } \\
\text { Diabetes }\end{array}$ \\
FBG & Fasting blood glucose \\
FPG & Fasting plasma glucose \\
RARV & Residual absolute real variability \\
RSD & Residual standard deviation \\
UKPDS & UK Prospective Diabetes Study \\
VADT & Veteran Affairs Diabetes Trial
\end{tabular}

To the Editor: Trials of intensive glucose lowering in type 2 diabetes have yielded mixed results for the effectiveness of this approach on microvascular complications [1]. Recent post hoc analyses of several trials have indicated that glucose variability may influence these same outcomes and help to explain the discrepancy among trial results [2]. The relevance of glucose variability, however, has not been examined carefully across a wide variety of glucose-lowering trials in type 2 diabetes, particularly in those enrolling people with new-

Electronic supplementary material The online version of this article (https://doi.org/10.1007/s00125-020-05273-7) contains peer-reviewed but unedited supplementary material, which is available to authorised users.

Jin J. Zhou

jzhou@email.arizona.edu

1 Department of Epidemiology and Biostatistics, Mel and Enid Zuckerman College of Public Health, University of Arizona, Tucson, AZ, USA

2 Carl T. Hayden Phoenix VA Health Care System (111E), Phoenix, AZ, USA

3 Diabetes Trials Unit, Oxford Centre for Diabetes, Endocrinology and Metabolism, University of Oxford, Oxford, UK onset type 2 diabetes. Here we evaluate the impact of glucose variability on nephropathy risk in three major and welldescribed glucose-lowering trials: the UK Prospective Diabetes Study (UKPDS) [3], the Action to Control Cardiovascular Risk in Diabetes (ACCORD) trial [4] and the Veteran Affairs Diabetes Trial (VADT) [5]. Using similar eGFR definitions for each study, we performed a metaanalysis to aggregate evidence from all three trials.

UKPDS (ISRCTN Registry identifier: ISRCTN75451837) was designed to establish whether intensive blood-glucose control reduced the risk of macrovascular or microvascular complications in individuals with type 2 diabetes. UKPDS participants all had newly diagnosed type 2 diabetes, with a mean age of 53 years [3] (see electronic supplementary material [ESM] Table 1 for participant baseline characteristics). They were randomised to three treatment strategies: conventional (primarily with diet), intensive (using insulin or sulfonylureas) and intensive (using metformin in overweight participants). Fasting plasma glucose (FPG) was measured every 3 months over the first 5 years of follow-up. On-study median (IQR) FPG values (mmol/l) after 3 months for the three treatment arms were 8.5 (7-10.7), 7.0 (5.9-8.6) and 7.5 (6.4-9.2), respectively (ESM Fig. 1a).

ACCORD (ClinicalTrials.gov registration no. NCT00000620) was a double two-by-two factorial, parallel treatment trial in which individuals were randomised to receive intensive or standard glucose-lowering treatments, as well as to distinct blood pressure and lipid interventions arms [6] (see ESM Table 2 for baseline characteristics of participants). It included participants with a mean age of 63 years, mean type 2 diabetes duration of 12 years and $\mathrm{HbA}_{1 \mathrm{c}}$ concentrations $\geq 58.5 \mathrm{mmol} / \mathrm{mol}(7.5 \%)$. During the study, fasting blood glucose (FBG) was measured every 4 months, up to a maximum of 84 months. After 6 months, median (IQR) FBG concentrations $(\mathrm{mmol} / \mathrm{l})$ for the standard and intensive 
treatment groups were $8.4(6.8-10.2)$ and $6.4(5.2-8.3)$, respectively (averaged over all time points after 6 months), and were subsequently generally maintained in this range (ESM Fig. 1c).

VADT (ClinicalTrials.gov registration no. NCT00032487) randomised military veterans with a mean age of 60 years and mean type 2 diabetes duration of 12 years, with suboptimal response to therapy for type 2 diabetes, to receive either intensive or standard glucose control [5] (ESM Table 3). During the study, FBG was measured every 4 months, up to a maximum of 84 months. After 6 months, median (IQR) FBG concentrations $(\mathrm{mmol} / \mathrm{l})$ were stabilised for the standard and intensive treatment arms at $9(7.1-11.3)$ and $6.5(5.1-8.4)$, respectively (averaged over all time points after 6 months; ESM Fig. 1d).

As the trials included individuals at different disease stages at enrolment, we defined our primary nephropathy outcome as at least two consecutive post randomisation visits with eGFR $<45 \mathrm{ml} \mathrm{min}{ }^{-1}[1.73 \mathrm{~m}]^{-2}$ to obtain a sufficient number of events. We excluded participants with less than two eGFR measurements or if eGFR was consistently $<45 \mathrm{ml} \mathrm{m^{-1 }}$ $[1.73 \mathrm{~m}]^{-2}$. For this analysis, FPG observations from the first 3 months of the UKPDS, first 4 months of ACCORD and first
6 months of VADT were excluded to eliminate the effect of rapid reduction in FPG (which varied per protocol, across trials) on glycaemic variation measures during the early trial periods. This left 4185 of 4209 participants from the UKPDS, 9970 of 10,251 participants from ACCORD, and 1606 of 1791 from VADT. This study has the approval of the local ethics committee.

The monotonic increase in mean FPG over time in the UKPDS cohort (ESM Fig. 1a), leads to overestimates of glycaemic variability with large SD values. Given the approximately linear FPG increase $(0.02 \mathrm{mmol} / \mathrm{l})$ per month (standard error, defined as SD of the estimated regression coefficient, is $0.01 ; p<0.0001$ for linear trend), the slope was estimated using a linear mixed-effects model with random intercept and analysis of residuals for model diagnosis.

Glucose variability can be estimated using residuals around its linear trend against time [7] and largely eliminates the effect of changing ambient glucose control (see ESM Fig. 1b for residual FPG values in UKPDS). Residual standard deviation (RSD) and residual absolute real variability (RARV) were used to measure glycaemic variability across all studies for

Table 1 HR of residual glucose variability for nephropathy

\begin{tabular}{|c|c|c|c|c|c|c|}
\hline \multirow[t]{2}{*}{$\begin{array}{l}\text { Measure of glycaemic } \\
\text { variability }\end{array}$} & \multicolumn{2}{|l|}{$\begin{array}{l}\text { Model } 1 \\
\text { (age adjustment) }\end{array}$} & \multicolumn{2}{|c|}{$\begin{array}{l}\text { Model } 2^{\mathrm{a}} \\
\text { (multivariate adjustment) }\end{array}$} & \multicolumn{2}{|c|}{$\begin{array}{l}\text { Model } 3^{\mathrm{b}} \\
\left(\text { Model } 2+\text { cumul. average } \mathrm{HbA}_{1 \mathrm{c}}\right)\end{array}$} \\
\hline & HR $(95 \%$ CI $)$ & $p$ value & HR $(95 \%$ CI $)$ & $p$ value & HR $(95 \%$ CI) & $p$ value \\
\hline \multicolumn{7}{|l|}{ UKPDS $(n=4185)$} \\
\hline RSD & $1.49(0.90,2.49)$ & 0.120 & $1.86(1.07,3.26)$ & 0.027 & $1.74(0.84,3.61)$ & 0.133 \\
\hline RARV & $1.39(0.83,2.30)$ & 0.219 & $1.71(0.99,2.96)$ & 0.056 & $1.66(0.81,3.44)$ & 0.168 \\
\hline \multicolumn{7}{|l|}{$\operatorname{ACCORD}(n=9930)$} \\
\hline RSD & $1.56(1.39,1.76)$ & $<0.0001$ & $1.46(1.29,1.67)$ & $<0.0001$ & $1.37(1.18,1.59)$ & $<0.0001$ \\
\hline RARV & $1.40(1.29,1.54)$ & $<0.0001$ & $1.34(1.21,1.50)$ & $<0.0001$ & $1.27(1.13,1.43)$ & $<0.0001$ \\
\hline \multicolumn{7}{|l|}{$\operatorname{VADT}(n=1606)$} \\
\hline RSD & $1.86(1.50,2.30)$ & $<0.0001$ & $1.54(1.23,1.93)$ & $<0.0001$ & $1.57(1.22,2.03)$ & 0.002 \\
\hline RARV & $1.66(1.41,1.97)$ & $<0.0001$ & $1.45(1.20,1.74)$ & $<0.0001$ & $1.47(1.19,1.80)$ & 0.001 \\
\hline \multicolumn{7}{|c|}{ Meta-analysis $^{\mathrm{c}}(n=15,753)$} \\
\hline RSD & $1.61(1.46,1.79)$ & $<0.0001$ & $1.49(1.33,1.66)$ & $<0.0001$ & $1.40(1.24,1.59)$ & $<0.0001$ \\
\hline RARV & $1.45(1.35,1.58)$ & $<0.0001$ & $1.37(1.25,1.50)$ & $<0.0001$ & $1.31(1.18,1.44)$ & $<0.0001$ \\
\hline
\end{tabular}

eGFR was generated from the Modification of Diet in Renal Disease Study (MDRD) equation for each study. Nephropathy was defined as eGFR $<45 \mathrm{ml} \mathrm{min}{ }^{-1}[1.73 \mathrm{~m}]^{-2}$

${ }^{a}$ Model 2: in each study, Model 2 is adjusted for all covariates that significantly differed between those with and without nephropathy during the study (ESM Table 1). In UKPDS, in addition to baseline age, we additionally adjusted for: baseline diastolic blood pressure (DBP), systolic blood pressure (SBP), LDL, total cholesterol, baseline eGFR, Early Treatment Diabetic Retinopathy Study (ETDRS) test score prior to randomisation, and sex. In ACCORD, in addition to baseline age, we additionally adjusted for: duration of diabetes, baseline DBP, SBP, HDL, total cholesterol, eGFR, sex, race, CVD history, and history of heart failure and eye disease. In VADT, we additionally adjusted for diabetes duration, SBP, DBP, baseline eGFR, and history of CVD and eye disease. Note: since eGFR was estimated using creatine levels, we did not adjust for albumin/creatinine ratio (ACR) even though it was a significantly different covariate between those with and without nephropathy (ESM Table 2 and ESM Table 3)

${ }^{\mathrm{b}}$ Model 3: additionally, adjusted for cumulative (cumul.) average of $\mathrm{HbA}_{1 \mathrm{c}}$ as well as the covariates adjusted for in Model 2

${ }^{\mathrm{c}}$ A fixed-effect inverse variance meta-analysis was adopted and $Q$ heterogeneity statistics were all non-significant $(>0.05)$ (a random-effect metaanalysis yielded very similar results) 
consistency. RSD and RARV were calculated as follows:

$\mathrm{RSD}=\sqrt{\frac{\sum_{i=1}^{n}\left(x_{i}-\widehat{x}_{i}\right)^{2}}{n-2}}$

$\mathrm{RARV}=\frac{1}{n-1} \sum_{i=1}^{n-1}\left|\left(x_{i+1}-\widehat{x}_{i+1}\right)-\left(x_{i}-\widehat{x}_{i}\right)\right|$

where $\left(\widehat{x}_{1}, \widehat{x}_{2}, \cdots, \widehat{x}_{n}\right)$ are the fitted values from a linear mixedeffect model with time measured by month as fixed effect and a random intercept. In these formulas, $n$ is the total number of measures for an individual, while $i$ is the indexing for each measure.

Multivariable analyses were performed using Cox proportional hazard models to evaluate the time-dependent effects of glucose variability measures. Statistical analyses, including a fixed-effects meta-analysis, were conducted by $\mathrm{R}$ analysis software (The R Foundation for Statistical Computing, version 3.6.3; www.r-project.org. Package 'coxph' version 3.2-3 was adopted for cox proportional hazard model analysis and package 'nlme' version 3.1-148 was adopted for longitudinal FPG modelling). A two-sided $p$ value $<0.05$ was considered statistically significant.

In the age-adjusted model (Model 1; Table 1), we show a substantial increased risk for nephropathy with increasing RSD or RARV in all three studies, with estimated HR ranging from 1.39 to 1.86 . However, although relatively similar HRs were seen in the UKPDS as compared with the other studies, these did not reach statistical significance, likely due to fewer events. After adjusting for differences in baseline significant nephropathy risk factors (Model 2; Table 1), risk of RSD and RARV in both ACCORD and VADT remained significant, with RSD also becoming significant in UKPDS. After further adjustment for cumulative $\mathrm{HbA}_{1 \mathrm{c}}$ as a reflection of average glycaemic control (Model 3; Table 1), HRs were only modestly changed in all studies. Results were similar if additionally adjusting for treatment assignment. Our meta-analysis of these three studies shows that glycaemic variability (measured by both RSD and RARV) is associated with a $30-40 \%$ increase (meta-analysis data for Model 3; Table 1) in the risk of developing moderate to severe nephropathy (eGFR $<45 \mathrm{ml} \mathrm{min}^{-1}$ $[1.73 \mathrm{~m}]^{-2}$ ) for each 1-unit increment in RSD or RARV, which was independent of overall average glycaemic control.

The current results demonstrate a consistent finding that glycaemic variability in the setting of efforts to lower overall glucose control is associated with increased risk for moderate to severe nephropathy. Results from several previous investigations of longterm glycaemic variability and nephropathy are generally supportive of our findings [8]. Our analysis is based on three large and well-designed clinical trials; with larger sample sizes than previously published trial design reports, it substantially bolsters the support for the concept that long-term glucose variability poses a risk for nephropathy complications among individuals with diabetes. Importantly, participants in our analysis represented individuals with a broad range of type 2 diabetes disease stages and cardiovascular risk history, making our findings more generalisable.

Data availability Individual deidentified participant data of ACCORD was requested through BioLINCC (https://biolincc.nhlbi.nih.gov/ home/). The independent scientific review board reviewed and approved the request. The study protocols are available on ClinicalTrials.gov. VADT and UKPDS deidentified participant data will not be available online, but upon request.

Funding This work was supported by the Veterans Affairs Cooperative Studies Program, Department of Veterans Affairs Office of Research and Development. Additional support was received from the National Institutes of Health R01-067690 and 5R01- 094775 to PR, and the American Diabetes Association to PR. JJZ is supported by the NIH grant K01DK106116 and an Arizona Biomedical Research Centre (ABRC) new investigator award. RRH is an emeritus UK National Institute for Health Research Senior Investigator.

Authors' relationships and activities The authors declare that there are no relationships or activities that might bias, or be perceived to bias, their work.

Contribution statement JJZ and PR conceived the idea. JJZ conducted the analysis and drafted the manuscript. RC and RRH provided UKPDS data. PR provided VADT data. All authors helped to interpret the data and write the manuscript. JJZ is the guarantor of this work. All authors provided final approval of the version to be published.

\section{References}

1. Zoungas S, Arima H, Gerstein HC et al (2017) Effects of intensive glucose control on microvascular outcomes in patients with type 2 diabetes: a meta-analysis of individual participant data from randomised controlled trials. Lancet Diabetes Endocrinol 5(6):431437. https://doi.org/10.1016/S2213-8587(17)30104-3.

2. Hirakawa $Y$, Arima H, Zoungas S et al (2014) Impact of visit-to-visit glycemic variability on the risks of macrovascular and microvascular events and all-cause mortality in type 2 diabetes: the ADVANCE trial. Diabetes Care 37(8):2359-2365. https://doi.org/10.2337/dc140199

3. UK Prospective Diabetes Study (UKPDS) Group (1998) Intensive blood-glucose control with sulphonylureas or insulin compared with conventional treatment and risk of complications in patients with type 2 diabetes (UKPDS 33). Lancet 352(9131):837-853. https:// doi.org/10.1016/S0140-6736(98)07019-6

4. The Action to Control Cardiovascular Risk in Diabetes Study Group (2008) Effects of intensive glucose lowering in type 2 diabetes. $\mathrm{N}$ Engl J Med 358(24):2545-2559. https://doi.org/10.1056/ NEJMoa0802743

5. Duckworth W, Abraira C, Moritz T et al (2009) Glucose control and vascular complications in veterans with type 2 diabetes. $\mathrm{N}$ Engl $\mathrm{J}$ Med 360(2):129-139. https://doi.org/10.1056/NEJMoa0808431

6. Ismail-Beigi F, Craven T, Banerji MA et al (2010) Effect of intensive treatment of hyperglycaemia on microvascular outcomes in type 2 diabetes: an analysis of the ACCORD randomised trial. Lancet 376(9739):419-430. https://doi.org/10.1016/S0140-6736(10) 60576-4. 
7. Diaz KM, Tanner RM, Falzon L et al (2014) Visit-to-visit variability of blood pressure and cardiovascular disease and all-cause mortality: a systematic review and meta-analysis. Hypertension 64(5):965982. https://doi.org/10.1161/HYPERTENSIONAHA.114.03903.

8. Gorst C, Kwok CS, Aslam S et al (2015) Long-term glycemic variability and risk of adverse outcomes: a systematic review and meta- analysis. Diabetes Care 38(12):2354-2369. https://doi.org/10.2337/ dc15-1188

Publisher's note Springer Nature remains neutral with regard to jurisdictional claims in published maps and institutional affiliations. 